Research Paper

\title{
Theranostic Oxygen Delivery Using Ultrasound and Microbubbles
}

\author{
James J. Kwan ${ }^{1}$, Mehmet Kaya ${ }^{2,3}$, Mark A. Borden ${ }^{1}$ and Paul A. Dayton ${ }^{2}$ \\ 1. Department of Mechanical Engineering, University of Colorado, Boulder, CO 80309 \\ 2. Joint Department of Biomedical Engineering, University of North Carolina and North Carolina State University, Chapel Hill, NC 27599 \\ 3. (new affiliation) Department of Biomedical Engineering, Florida Institute of Technology, Melbourne, FL 32901
}

$\bowtie$ Corresponding author: Paul A. Dayton, Ph.D. Biomedical Engineering. 152 MacNider Hall, CB 7575, Chapel Hill, NC, 27599. padayton@bme.unc.edu. phone: (919) 843 9521. fax: (919) 8439520

(C) Ivyspring International Publisher. This is an open-access article distributed under the terms of the Creative Commons License (http://creativecommons.org/ licenses/by-nc-nd/3.0/). Reproduction is permitted for personal, noncommercial use, provided that the article is in whole, unmodified, and properly cited.

Received: 2012.03.08; Accepted: 2012.06.08; Published: 2012.12.23

\begin{abstract}
Means to overcome tumor hypoxia have been the subject of clinical investigations since the 1960's; however these studies have yet to find a treatment which is widely accepted. It has been known for nearly a century that hypoxic cells are more resistant to radiotherapy than aerobic cells, and tumor hypoxia is a major factor leading to the resistance of tumors to radiation treatment as well as several cytotoxic agents. In this manuscript, the application of ultrasound combined with oxygen-carrier microbubbles is demonstrated as a method to locally increase dissolved oxygen. Microbubbles can also be imaged by ultrasound, thus providing the opportunity for image-guided oxygen delivery. Simulations of gas diffusion and microbubble gas exchange show that small amounts (down to 5 vol\%) of a low-solubility osmotic gas can substantially increase microbubble persistence and therefore production rates and stability of oxygen-carrier microbubbles. Simulations also indicate that the lipid shell can be engineered with long-chain lipids to increase oxygen payload during in vivo transit. Experimental results demonstrate that the application of ultrasound to destroy the microbubbles significantly enhances the local oxygen release. We propose this technology as an application for ultrasound image-guided release of oxygen directly to hypoxic tissue, such as tumor sites to enhance radiotherapy.
\end{abstract}

Key words: Hypoxia, Tumor, Radiotherapy, Oxidation, Oxygenation

\section{Introduction}

Tumors are often characterized by lowered vascular density, irregular vascular geometry, inconsistent oxygen gradients and rheologic effects which contribute to hypoxic conditions [1]. It has been known for nearly a century that hypoxic cells are more resistant to radiotherapy than aerobic cells, and tumor hypoxia is a major factor leading to the resistance of tumors to radiation treatment and cytotoxic agents [2,3]. Additionally, tumor hypoxia is associated with increased likelihood of regional recurrence and metastases, and often leads to poor treatment outcome and prognosis [2, 4-7].
Means to overcome tumor hypoxia have been the subject of clinical investigations since the 1960's; these studies, however, have yet to find a treatment that is widely accepted. Technical logistics limit the practical use of hyperbaric oxygen delivery, inhaled carbogen has been shown to result in vasoconstriction which counteracts the desired effect, and nitroimidazoles and other radiosensitizers have not gained favor because of their toxicity and lack of consistent effectiveness [8]. Yet scientists believe "there is good evidence ... that improving clinical outcome by hypoxic modulation is an achievable goal" [8]. 
In a review by Brown and Wilson [9], it was reported that all human tumors have median oxygen levels significantly lower than their normal tissue of origin. Unlike the normal subcutaneous tissue that has a median oxygen partial pressure between 40 and $60 \mathrm{mmHg}$, many tumors have an oxygen partial pressure on the order of $10 \mathrm{mmHg}$ [9]. Full resistance to radiation occurs when the cells are near anoxic, with very low oxygen levels of approximately 0.2 $\mathrm{mmHg}$; however, resistance to radiation begins at higher oxygen concentrations of about $20 \mathrm{mmHg}$ [9]. Therefore, the oxygen partial pressures between 0.2 and $20 \mathrm{mmHg}$ can be described as an intermediate range of resistance to radiation.

A radiation survival curve illustrates the surviving fraction of tumor cells with respect to radiation dose, where the effect of oxygen is to reduce the slope of this curve. The "oxygen enhancement ratio" (OER) is often described as the degree of radiation sensitization and refers to the ratio of doses in the absence of oxygen and in the presence of oxygen to provide the same cell survival. The value for mammalian cells was given to be between 2.5 to 3.0. This value includes several endpoints including cell killing, mutagenesis, and the induction of chromosome aberrations [10]. The seemingly low OER value of 2.6 corresponds to $50 \%$ of cells killed under hypoxic conditions as opposed to almost $99 \%$ of the cells killed under aerobic conditions with a radiation dose of 1,000 cGy [11]. This information implies that tumor cells with oxygen levels in the intermediate range can be re-oxygenated, which would result in an increased sensitivity of cells to radiation. Additionally, it has been demonstrated [12] that hypoxic tumor cells become sensitive for radiation even if the oxygen is present less than a second before or after exposure to radiation therapy.

More recent work has correlated low reactive oxygen species (ROS) concentrations with radioresistance in cancer stem cells [13]. Pharmacological depletion of ROS scavengers in cancer stem cells was shown to decrease their clonogenicity and result in radiosensitization. An alternative approach may be to increase local oxygen concentration, and therefore ROS production, in order to improve ionizing-radiation-induced cell killing of cancer stem cells and their tumorigenic progeny.

Ultrasound, in conjunction with microbubbles, has the unique advantage of being able to provide safe, noninvasive and image-guided, site-specific treatment. An ultrasound transducer can be used to image the local anatomy in order to define the region for treatment. Microbubbles are highly echogenic intravascular contrast agents that can be used to help define the vascular architecture and blood flow pat- terns in the tumor area $[14,15]$. Targeted microbubbles can be used to image endothelial epitopes associated with neovascularization and inflammation, thereby enabling ultrasound molecular imaging [16-18]. Ultrasound also applies the required therapeutic pulses to the sample volume by focusing on the tumor region with imaging guidance for feedback. Microbubbles have already been FDA approved in the US for contrast agents in echocardiography, and imaging microbubbles are used even more extensively in Canada, Europe, India and Asia. Therapeutic microbubbles for oxygen delivery could be made with similar characteristics.

Although a few prior studies have investigated the use of microbubbles for the delivery of oxygen, these studies have largely focused on microbubbles to be injected into the circulation to increase systemic oxygen for anemia [19-23]. Burkard and Van Liew [24] proposed that microbubbles containing a heavy gas (osmotic agent) could serve as an blood substitute. In an animal study by Lundgren et al. [21], it was suggested that an emulsion of dodecafluoropentane (DDFP) injected intravascularly combined with oxygen breathing could transfer physiologically significant amounts of oxygen in severely anemic rats. Gerber et al. [19] examined the stability of microbubbles made with fluorinated phosholipid instead of a standard hydrogenated phospholipid shell. They suggested that microbubbles could be engineered to comprise a shell and gas core to promote a longer in vivo half-life and more efficacious systemic delivery. To our knowledge, however, the application of ultrasound for targeted oxygen delivery to overcome local hypoxia has not yet been demonstrated.

The use of ultrasound in our study as a mechanism for local microbubble fragmentation makes it possible for the oxygen to be released from the microbubbles directly at the tumor site right before or even during radiation therapy. In this manner, oxygen is released immediately in tumor microvasculature rather than slowly and throughout the entire systemic circulation, as would be the case with non-ultrasound enhanced mechanisms. Hence, we hypothesize that ultrasound combined with oxygen delivery microbubbles can provide a means of greatly enhancing the tumor-specific dose of oxygen. Additionally, we propose that the already demonstrated contrast enhancement capabilities of oxygen microbubbles can be used to guide and monitor the ultrasound-mediated oxygen release in situ. In this manuscript, we perform theoretical calculations to investigate oxygen $\mathrm{mi}-$ crobubble stability and experimentally demonstrate the ability of ultrasound to locally enhance oxygen concentration through the disruption of oxy- 
gen-carrier microbubbles.

\section{Theory}

Fluorocarbon gases have low aqueous solubility and diffusivity, and thus have been used as osmotic agents to stabilize oxygen-carrier microbubbles [24, 25]. We employed a multi-gas microbubble dissolution model to elucidate the role of the fluorocarbon on microbubble stability and oxygen release $[26,27]$. The model describes the transient change in gas-core contents and microbubble size as a function of the microbubble shell properties and surrounding gas composition. The model includes a coupled set of nonlinear differential equations describing the gas accumulation, which is coupled to both the mechanical properties and the gas permeability of the microbubble shell:

$$
\begin{gathered}
\frac{-d n_{i}}{d t}= \\
\frac{4 \pi R^{2} K_{H, i}}{\frac{R}{D_{i}}+\Omega_{s, i}}\left(\frac{2 \sigma}{R}+P_{H}-\frac{3 B T}{4 \pi R^{3}} \sum n_{j}-f_{i} P_{\text {sat }, i}\right) \text { where } j \neq i \\
\ldots(1) \\
0=2 \sigma R^{2}+P_{H} R^{3}-\frac{4 \pi}{3 B T} \sum n_{i} \quad \ldots(2)
\end{gathered}
$$

where $i$ represents a gas species $(j \neq i$ to prevent double counting), $n$ is the moles of gas in the microbubble core, $t$ is time, $R$ is the radius of the microbubble, $K_{H}$ is the Henry's constant, $D$ is the diffusivity of gas in the surrounding medium, $\Omega_{\mathrm{S}}$ is the resistance to gas permeation of the lipid shell, $\sigma$ is the surface tension (assumed to be isotropic), $P_{H}$ is the hydrostatic pressure, $B$ is the ideal gas constant, $T$ is the temperature, $f$ is the fraction of gas in surrounding bulk medium to that at saturation, and $P_{\text {sat }}$ is the saturation pressure of gas in the surrounding bulk medium. One can estimate the dependence of the gas permeability $\left(\Omega_{s}\right)$ by implementing an energy barrier model, as recently described by Kwan and Borden [27], which is shown below:

$$
\Omega_{s, i}=\Omega_{n} e^{\frac{\pi a_{i}^{2}}{k_{B} T}\left(\sigma_{0}-\sigma\right)}
$$

where $\Omega_{n}$ is an empirical constant dependent on the lipid encapsulation, $a$ is the collision radius of the gas molecule, $k_{B}$ is the Boltzmann constant, and $\sigma_{o}$ is the surface tension of a gas-liquid interface. Equation 1 is used to determine the flux of each gas into the microbubble over a given time step, and then equation 2 is used to determine the resulting microbubble radius. Numerical solution of the two equations provides a theoretical prediction of the microbubble radius-time behavior.

Previous experimental results showed that lipid-coated microbubbles experience a dynamic surface tension as they experience growth and compression during gas exchange [26, 27]. Based on the empirical constitutive developed model by Kwan and Borden [27], the radius-dependent surface tension is given by:

$$
\sigma(R)=\left\{\begin{array}{cc}
0 & R<R_{0}(\text { Resting }) \\
k_{E}\left(\left(\frac{R}{R_{0}}\right)^{2}-1\right) & 0<\sigma<\sigma_{\text {break }} \text { (Expansion) } \\
\sigma_{E Q}+\left(\sigma_{\text {break }}-\sigma_{E Q}\right) e^{-\kappa_{E}\left(t-t_{\text {break }}\right)} & R_{\text {break }}<R<R_{\text {max }} \text { (Expansion) } \\
\sigma_{R_{\text {max }}}+k_{C}\left(\frac{R^{2}-R_{\text {max }}{ }^{2}}{R_{0}{ }^{2}}\right) & R_{0}<R<R_{\text {max }} \text { (Compression) }
\end{array}\right.
$$

where $k_{E}$ is the linear expansion elasticity of the lipid monolayer, $R_{0}$ is the initial radius of the microbubble, $\sigma_{\text {break }}$ is the rupture tension of the lipid monolayer $(\sim 73 \mathrm{mN} / \mathrm{m}), \sigma_{E Q}$ is the equilibrium spreading tension $(\sim 25 \mathrm{mN} / \mathrm{m}), \kappa_{E}$ is the domain relaxation rate (a measure of domain dissolution) of the interface after rupture, $R_{\text {break }}$ is the radius at which rupture occurs, $R_{\max }$ is the maximum radius before dissolution, $\sigma_{R \max }$ is the tension of the monolayer at the maximum radius, and $k_{C}$ is the compression elasticity. This model was derived to explain the resistance towards expansion behavior observed by microscopy images of lipid-coated microbubbles undergoing gas exchange [27]. To our knowledge, this model most accurately accounts for the highly nonlinear properties of the lipid shell during the relatively slow dynamics associated with microbubble dissolution in vivo.

\section{Materials and Methods}

\section{Microbubble Formulation}

The lipids 1,2-distearoyl-sn-glycero-3phosphocholine (DSPC) and 1,2-distearoyl-snglycero-3-phosphoethanolamine-N-[methoxy(polye thyleneglycol)-2000] (DSPE-PEG2000) were purchased from Avanti Polar Lipids (Alabaster, AL, USA). The buffer solution for the lipids was prepared using Tris (Hydroxymethyl) Aminomethane pur- 
chased from EMD Chemicals (Gibbstown, NJ, USA). The $\mathrm{pH}$ of the buffer solution was adjusted to 7.4 using hydrochloric acid. The lipid composition included $1.5 \mathrm{mg} / \mathrm{mL}$ DSPC in 10 vol. \% glycerin (Fisher Scientific, Pittsburgh, PA, USA), 10 vol. \% propylene glycol (Sigma-Aldrich, St. Louis, MO, USA), and 80 vol. \% deionized water, with a 9:1 mol:mol ratio of DSPC to DSPE-PEG2000. The lipids dissolved in chloroform were exposed to nitrogen and degassed for 16 hours to evaporate the chloroform. Once the lipids were dry, the buffer solution was added to the lipids in a vial. The lipid solution was sonicated until a transparent and homogeneous mixture was achieved. Vials of 1.5 $\mathrm{mL}$ of the lipid solution were degassed for 5 minutes. Microbubble solutions were activated by shaking vials in a Vialmix shaker (Bristol-Myers Squibb Medical Imaging Inc., North Billerica, MA, USA) as previously described [28]. Concentration and size distribution of microbubble suspensions were determined with an optical particle counter with a $0.5 \mu \mathrm{m}$ diameter lower detection limit (Accusizer Particle Sizing Systems, Santa Barbara, CA, USA).

\section{Ultrasound Generation for Bubble Disruption}

A single element $1 \mathrm{MHz}$ unfocused ultrasound transducer (Valpey Fisher Corp., Hopkinton, MA, USA) was used for transmission. The transducer was excited with a 10 cycle sinusoid pulse with pulse repetition frequency of $25 \mathrm{~Hz}$. The pulse was generated on an arbitrary wave generator (AWG 2021, Tektronix, Inc., Beaverton, OR, USA) and amplified by an RF amplifier (3200 L, ENI, Rochester, NY, USA). A calibrated needle hydrophone (Onda Corp., Sunnyvale, CA, USA) was used to record acoustic pressure

measurements. The transducer focus occurred at 6.4 $\mathrm{mm}$ from the transducer face where the acoustic pressure was measured to be $1200 \mathrm{kPa}$ peak negative pressure. Prior studies have demonstrated that for a transducer with a center frequency of $1 \mathrm{MHz}$, acoustic pressures greater than $800 \mathrm{kPa}$ are sufficient to fragment lipid-encapsulated microbubbles [29]. Although we chose to use $1 \mathrm{MHz}$ in this study for convenience, it is important to note that higher frequencies and pressures, such as those regularly used in clinical imaging applications, could also be used to fragment the microbubbles and release their gas content.

\section{Dissolved Oxygen Measurements}

For dissolved-oxygen measurement experiments, a small amount of microbubbles was injected using a calibrated pipetter (Biohit, Neptune, NJ, USA) into $70 \mathrm{~mL}$ of deionized water in a glass beaker. A stir bar in the beaker was used to gently keep the solution mixed during experiments. Prior to bubble addition, dissolved oxygen measurements determined between $45 \%-60 \%$ saturation at room temperature, indicating the water was partially saturated. A FOXY-R Oxygen Sensor and USB fiber-optic spectrometer (Ocean Optics Inc., Dunedin, FL, USA) was used to measure the change in the oxygen concentration during ultrasound exposure. The tip of ultrasound transducer was immersed into the beaker for ultrasound administration. All experiments were performed at $22{ }^{\circ} \mathrm{C}$ and 1 atm.

\section{Simulations} $[26,27]:$

Equations 1 and 2 were discretized as follows

$$
\begin{gathered}
n_{i, \tau+1}=-\Delta t \frac{4 \pi R_{\tau}^{2} K_{H, i}}{\Omega_{s, i_{\tau}}+\frac{R_{\tau}}{D_{i}}}\left[\frac{2 \sigma_{\tau}\left(R_{\tau}\right)}{R_{\tau}}-P_{s a t, i} f_{i}+P_{H}-\frac{3 B T}{4 \pi R_{\tau}{ }^{3}} \sum n_{j_{\tau}}\right]+n_{i_{\tau}} \text { where } j \neq i \\
0=2 \sigma\left(R_{\tau+1}\right) R_{\tau+1}^{2}+P_{H} R_{\tau+1}{ }^{3}-\frac{3 B T}{4 \pi} \sum n_{i_{\tau+1}}
\end{gathered}
$$

where $\tau$ represents the time step. Equations 5 and 6 were constrained by the Kwan-Borden condition [27]:

$$
\sigma\left(R_{\tau+1}\right)=\left\{\begin{array}{cc}
0 \frac{m N}{m} & R_{\tau+1}<R_{0} \\
k_{E}\left(\left(\frac{R_{\tau+1}}{R_{0}}\right)^{2}-1\right) & 0<\sigma<\sigma_{\text {break }} \\
\sigma_{E Q}+\left(\sigma_{\text {break }}-\sigma_{E Q}\right) e^{-\kappa_{E}\left(t-t_{\text {break }}\right)} & R_{\text {break }}<R_{\tau+1}<R_{\text {max }} \text { (Expansion) } \\
\sigma_{R_{\text {max }}}+k_{C}\left(\frac{R_{\tau+1}{ }^{2}-R_{\text {max }}{ }^{2}}{R_{0}{ }^{2}}\right) & R_{0}<R_{\tau+1}<R_{\text {max }} \text { (Compression) }
\end{array}\right.
$$

Equation 5 was solved at the beginning of each time step. The results of equation 5 were used in equation 6 , which was solved using a minimization algorithm in Matlab R2010b (MathWorks, Inc., Natick, MA, USA). Table 1 and 2 give the parameters used in the simulations. 
Table I: Gas properties used in modeling microbubble gas exchange.

\begin{tabular}{|c|c|c|c|c|}
\hline Gas Properties & Oxygen & Nitrogen & PFB & $\begin{array}{l}\text { Water } \\
\text { Vapor }\end{array}$ \\
\hline $\begin{array}{l}\text { Diffusivity }-\left[\mathrm{m}^{2} / \mathrm{s}\right] \mathrm{x} \\
10^{9}\end{array}$ & $3.948 \mathrm{a}, \mathrm{b}$ & $1.713 \mathrm{a}, \mathrm{b}$ & $0.4935^{b}$ & 315.16 \\
\hline $\begin{array}{l}\text { Henry's Constant - } \\
{\left[\left(\mathrm{g} / \mathrm{m}^{3}\right) / \mathrm{Pa}\right] \times 10^{4}}\end{array}$ & $2.42^{c, d}$ & $2 c, d$ & $0.69 \mathrm{~b}$ & $2.1^{*}$ \\
\hline Collision Diameter $[\AA ̊]$ & $0.34 \mathrm{e}$ & 0.37 e & $0.60 \mathrm{e}$ & $0.34 \mathrm{e}$ \\
\hline
\end{tabular}

Table 2: Lipid shell parameters used in modeling microbubble gas exchange.

\begin{tabular}{lllll}
\hline Lipid Properties & DMPC & DPPC & DSPC & DBPC \\
\hline $\begin{array}{l}\text { Expansion Elasticity - } \\
\text { [N/m] }\end{array}$ & $5.0^{\mathrm{f}}$ & $6.0^{\mathrm{f}}$ & $3.6^{\mathrm{f}}$ & $3.4^{\mathrm{f}}$ \\
$\begin{array}{l}\text { Domain Relaxation } \\
\text { Rate - [1/s] }\end{array}$ & $0.8^{\mathrm{f}}$ & $0.6^{\mathrm{f}}$ & $0.2^{\mathrm{f}}$ & $0.1^{\mathrm{f}}$ \\
$\begin{array}{l}\text { Compression Elasticity } \\
-[\mathrm{N} / \mathrm{m}]\end{array}$ & $0.07^{\mathrm{f}}$ & $0.3^{\mathrm{f}}$ & $0.3^{\mathrm{f}}$ & $0.7^{\mathrm{f}}$ \\
\hline $\mathrm{f}-[27]$ & & & & \\
\hline
\end{tabular}

\section{Results}

\section{Fabrication of stable, high-oxygen microbub- bles}

In initial studies, we attempted to formulate microbubbles with $100 \%$ oxygen [22, 23]. We observed, however, that the stability and production yield of the microbubbles was insufficient for ultrasound-mediated delivery (data not shown). Previous research has shown that use of a sparingly soluble "trapped species" can significantly enhance emulsion stability, presumably owing to Raoult's law where the chemical potential gradient for oxygen to diffuse into the microbubble in order to dilute the osmotic agent balances the chemical potential gradient for oxygen to dissolve out of the microbubble brought on by the capillary pressure $[35,36]$. We therefore hypothesized that the use of the perfluorobutane as a trapped species (osmotic agent) would increase the production yield and stability of the oxygen microbubbles without significantly affecting the oxygen loading capacity. To test this, we formulated the microbubbles with $95 \%$ oxygen and $5 \%$ perfluorobutane. The average microbubble diameter for the resulting oxygen/perfluorobutane mixture was measured to be approximately 4 microns, and the average microbubble concentration was on the order of $10^{10}$ per $\mathrm{mL}$ of suspension.

In order to confirm our experimental findings and provide a theoretical basis for the effect of the trapped species, we simulated microbubble stability using equations 5-6. The simulations predicted the dynamics of a newly generated microbubble submerged in a solution saturated with the filling gas (95\% oxygen and $5 \%$ perfluorobutane). The initial diameters of the microbubbles were set to 2, 4, 6 and $10 \mu \mathrm{m}$, and the surface tension was set to that of a clean gas/water interface $(73 \mathrm{mN} / \mathrm{m})$ to simulate a newly generated, uncoated microbubble. Figure 1 shows that the addition of perfluorobutane increased the dissolution time (increased stability) by an order of magnitude (11-fold) across all of the microbubble sizes. As expected, the PFB gas component behaved as an insoluble diluent and as a result reduced the overall efflux of gas out of the microbubble. Figure 1D indicates that the oxygen in the microbubbles quickly dissolves, concentrating the remaining PFB in the microbubble core. Consequently, the now predominately PFB gas core diffuses slowly, which can be seen as a shoulder on the diameter-time curves for the initially $\mathrm{O}_{2}$ :PFB-filled microbubbles (Figure 1B). Although these results neglect the effects of forming a lipid shell, they demonstrate that the longer residence time of the newly generated perfluorobutane-containing microbubbles allow for a significant increase in time for lipid adsorption, monolayer formation and stabilization through reduction in surface tension. Ultimately, the microbubble will equilibrate with the bulk concentration once Laplace pressure is removed [26, 27, 37-39].

\section{Ultrasound-Targeted Oxygen Release}

For the first part of the experiment, a fixed volume of $300 \mu \mathrm{L}$ of microbubbles $\left(95 \% \mathrm{O}_{2}\right.$ and $5 \%$ PFB) was injected into $70 \mathrm{~mL}$ of partially-saturated deionized water. The concentration of dissolved oxygen (given as percentage) was measured after 60, 120 and 240 seconds with and without ultrasound exposure. The values for the control group were obtained with microbubbles with the same mean population and size distribution without using the ultrasound. The rapid change in dissolved oxygen concentration was directly related to the application of ultrasound and increased with the duration of ultrasound exposure. As seen in Figure 2, after 240 seconds of ultrasound transmission, the change in the oxygen saturation was more than 3 times higher than the change in the oxygen when the solution was exposed to $60 \mathrm{sec}-$ onds of ultrasound. 

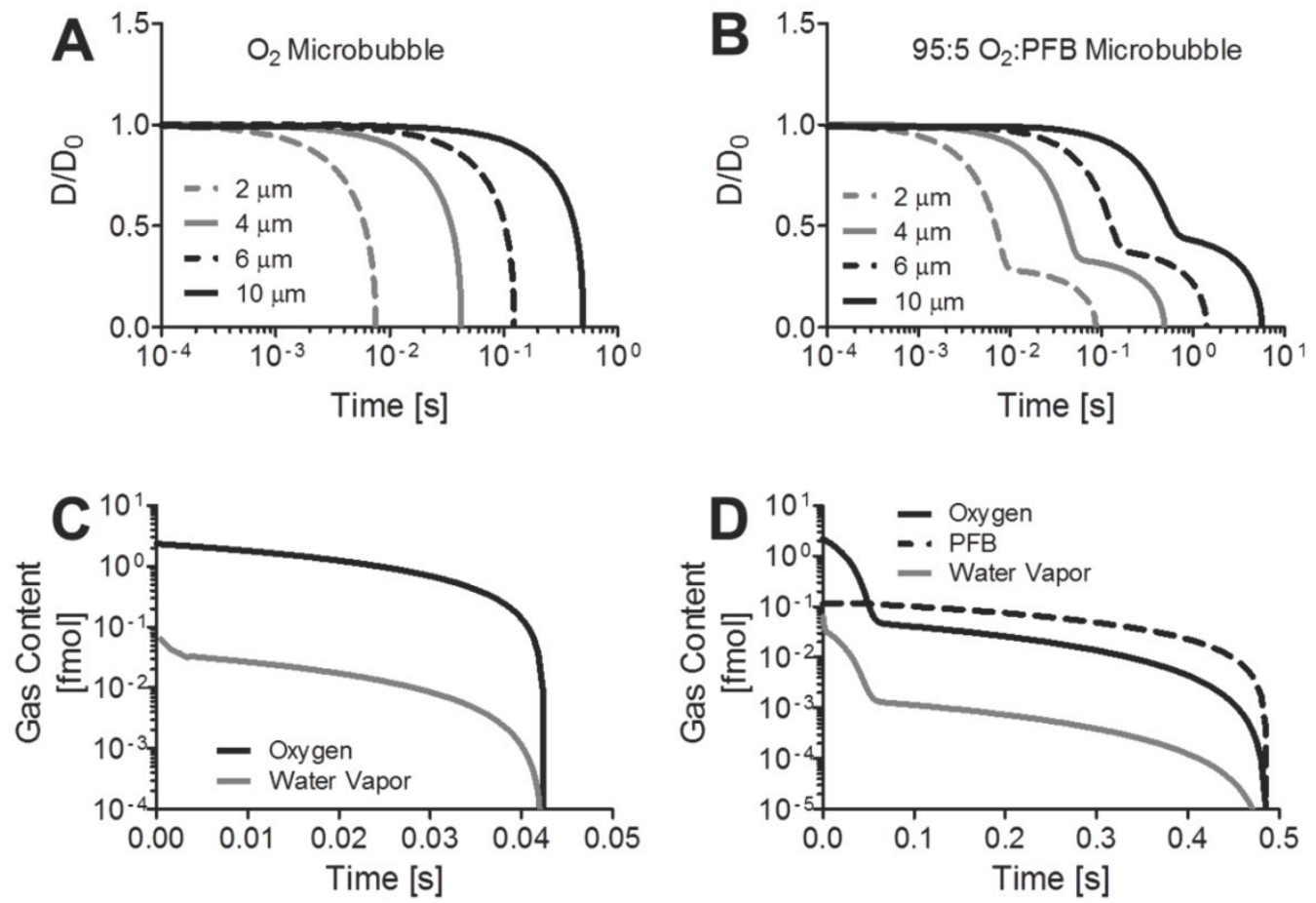

Figure I. Normalized diameter-time curves of uncoated, newly generated A) pure oxygen and B) $95 \%$ oxygen and $5 \%$ perfluorobutane microbubbles. Gas content over time of the initially generated C) pure oxygen and D) $95 \%$ oxygen and $5 \%$ perfluorobutane microbubbles. The initial diameters were set at 2, 4, 6, and $10 \mu \mathrm{m}$ and the surface tension was equal to a constant $73 \mathrm{mN} / \mathrm{m}$.

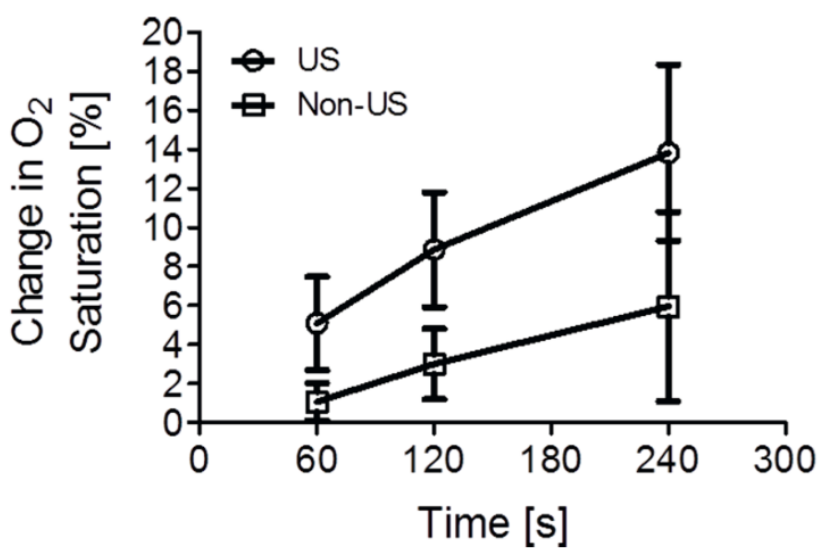

Figure 2. Effect of ultrasound exposure duration on oxygen release from microbubbles. Shown is the experimentally measured percent change in oxygen concentration following both ultrasound treated and untreated $300-\mu \mathrm{L}$ injection of $10^{10}$ microbubbles $/ \mathrm{mL}$ into $70-\mathrm{mL}$ of water.

The volume of bubbles added to the $70 \mathrm{~mL}$ of DI water was increased in steps of $100 \mu \mathrm{l}$, from 100 to 300 $\mu 1$. The dissolved oxygen concentration was measured after 60 and 120 seconds of ultrasound exposure. As in the previous part of the experiment, the control group was used to form a baseline with the same amount of microbubbles, but excluding ultrasound exposure.
Figure 3 shows the percent change in oxygen saturation as a function of microbubble concentration in 70 $\mathrm{mL}$ of partially saturated water, with and without ultrasound. Using the microbubble suspension in conjunction with ultrasound, we were able to achieve a significant $(p<0.05)$ increase in the dissolved oxygen content. The increase in percent oxygen saturation was $6 \%, 10 \%$ and $15 \%$ when $100 \mu \mathrm{L}, 200 \mu \mathrm{L}$ and $300 \mu \mathrm{L}$ of microbubbles were infused in the water.

Dissolved oxygen concentration was calculated as a function of bubble concentration and mean bubble size at $22{ }^{\circ} \mathrm{C}$ and $760 \mathrm{mmHg}$ total atmospheric pressure (see Appendix). At body temperature (37 $\left.{ }^{\circ} \mathrm{C}\right)$, the gas solubilities and diffusivities are slightly higher, and thus we expect a corresponding increase in the rate of gas exchange and oxygen release; however, this is not expected to change the conclusions drawn from this study. For an increase in temperature to Calculated values for dissolved oxygen concentration were compared with the measured values of percent dissolved oxygen saturation for increased volumes of microbubbles added to the solution as shown in Figure 4. The calculations were made for microbubbles between 100 and 300 microliters with 20 microliter intervals. The values for both calculated and experimental oxygen saturation were in agreement. 


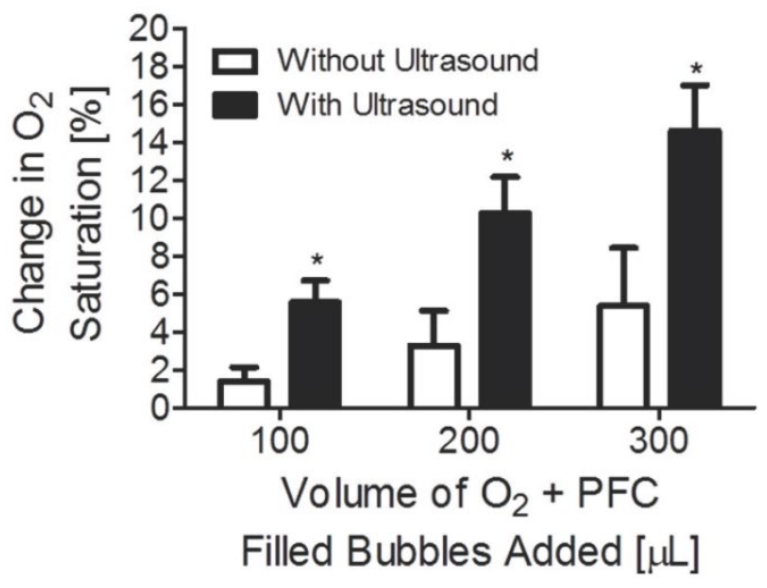

Figure 3. Percent change in oxygen saturation using microbubbles filled with oxygen and perfluorocarbon with and without ultrasound. The experimental values were obtained using 100 , 200 , and $300 \mu \mathrm{L}$ of microbubbles. The asterisk $(*)$ represents a one-tailed t-test $\mathrm{p}$ value below 0.05 .

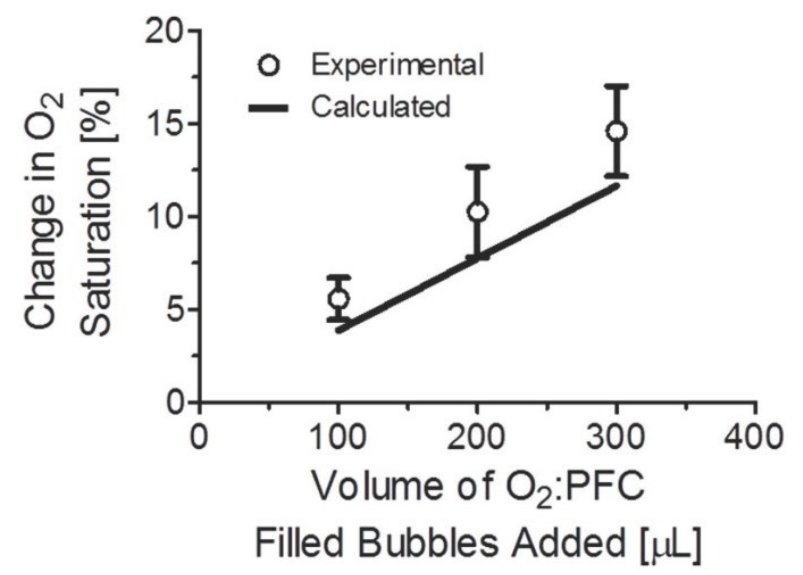

Figure 4. Percent change in oxygen saturation using microbubbles filled with oxygen and perfluorocarbon and calculated change in percent oxygen saturation. The predicted values were determined based on a 4- $\mu \mathrm{m}$ diameter bubble population with a concentration of $10^{10}$ bubbles $/ \mathrm{mL}$.

To demonstrate that the increase in oxygen saturation was primarily due to the activation of oxygen-PFC microbubble dissolution via ultrasound, the above study was also performed on microbubbles filled with nitrogen. As in the first part of the experiment, $300 \mu \mathrm{l}$ of nitrogen filled microbubbles were injected into $70 \mathrm{~mL}$ of DI water in a glass beaker. Dissolved oxygen concentration was measured after 60, 120 and 240 seconds of ultrasound exposure. The values for the control group, i.e., microbubbles not treated with ultrasound, were also obtained at the same time intervals. The increase in dissolved oxygen concentration was evident when we compared microbubbles filled with oxygen and PFC with microbubbles filled only with nitrogen. Figure 5 shows that microbubbles filled with nitrogen instead of oxygen have no significant effect on oxygen saturation.

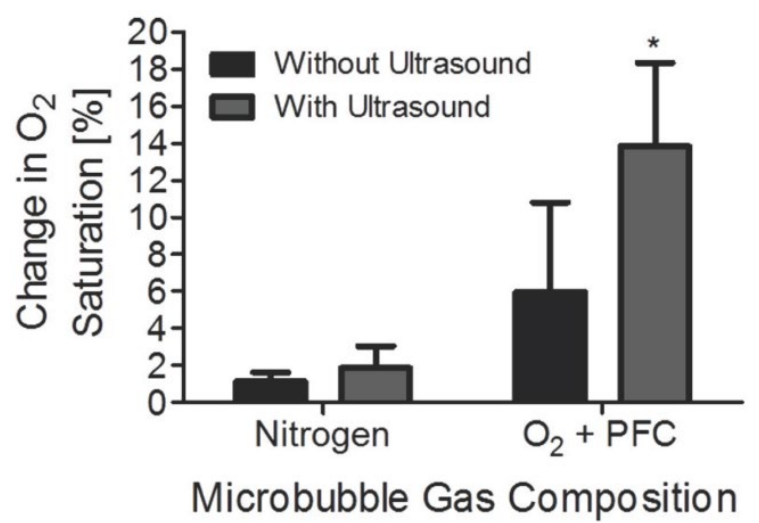

Figure 5. Comparison of mean percent change in oxygen saturation of $\mathbf{3 0 0}$ microliters of microbubbles filled with either pure nitrogen or with an oxygen and perfluorocarbon mixture. The time of ultrasound exposure was $240 \mathrm{~s}$. The asterisk $\left(^{*}\right)$ represents a one-tailed t-test $p$ value below 0.05 . Nitrogen-filled microbubbles did not increase oxygen saturation significantly.

\section{Simulations on microbubble persistence and oxygen payload during gas exchange}

Gas-exchange simulations of a lipid-encapsulated, 4- $\mu \mathrm{m}$ diameter microbubble suddenly submerged in an air-saturated solution were performed in order to mimic the experimental conditions. Results are shown in Figure 6 for the normalized diameter and the gas content in the microbubble. The lower partial pressure of oxygen in the surrounding milieu drives the efflux of oxygen, and the microbubble initially shrinks over the first one to ten seconds owing to the more rapid permeation of oxygen in water compared to nitrogen and the other gases. However, the partial pressure of nitrogen in the surrounding medium is higher, which drives its influx into the microbubble, and the radius increases back to the initial diameter. Nitrogen essentially displaces the excess oxygen and the microbubble rapidly becomes either an air bubble or a nitrogen bubble with the partial pressures of the contents equilibrating with the surrounding medium. Membrane elasticity prevents any expansion beyond the initial diameter. As a result, the final microbubble diameter is approximately equal to the initial diameter.

We also simulated the effect of the lipid shell composition on the transient oxygen gas payload. Interestingly, the interfacial mechanics of the mono- 
layer did not play a significant role; the microbubble did not present any significant growth and therefore did not leave the linear elastic regime of the monolayer. As a result, the shell composition did not influence the final microbubble size or core composition. The addition of methylene groups to the hydrophobic tail did however increase the overall time for gas exchange to take place. According to these simulations, this increase in duration for gas exchange was primarily due to the decrease in gas shell permeability for the larger acyl-chain lipids. For example,
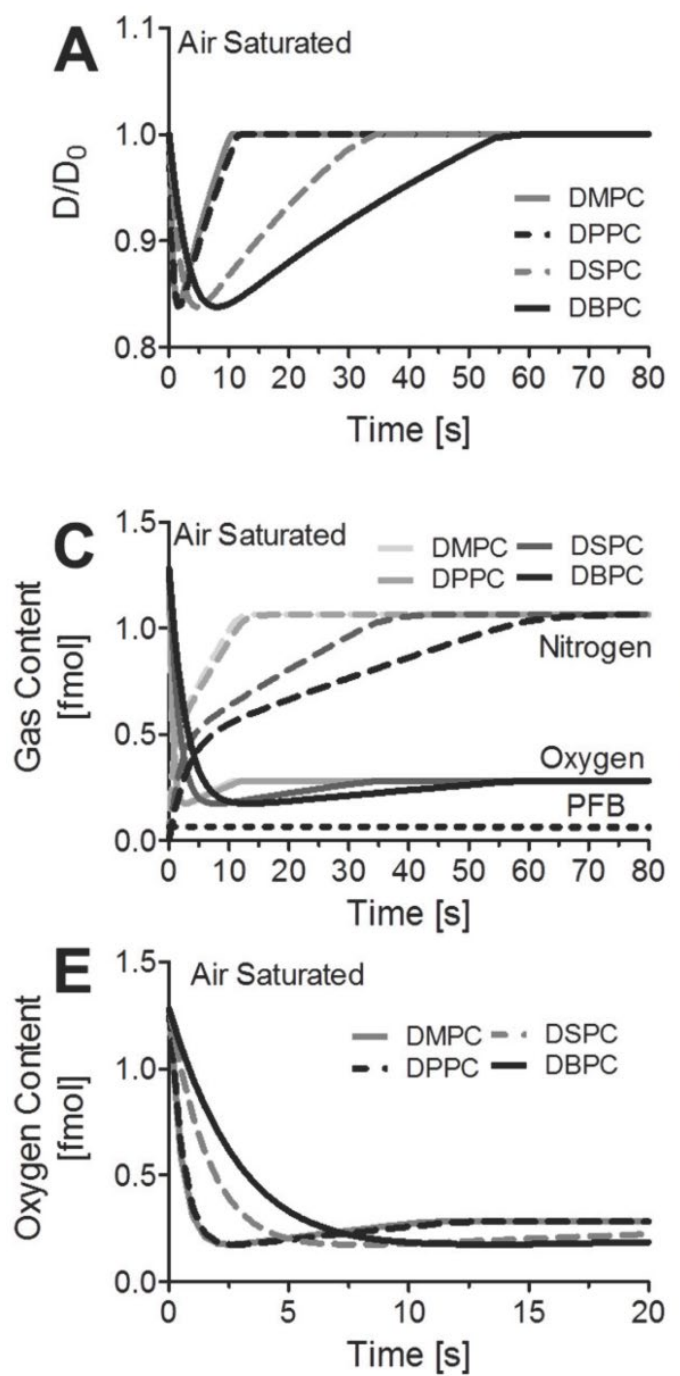

complete gas exchange in an air-saturated solution for DSPC- and DBPC-coated microbubbles was estimated to take 6 times longer than DMPC- and DPPC-coated microbubbles. Similarly, intravenously injected DBPC-coated oxygen microbubbles will deposit $\sim 99.9 \%$ of their payload in $\sim 15$ seconds compared to $\sim 3$ seconds for DMPC-coated microbubbles. Our predictions imply that the use of longer acyl-chain lipid encapsulations significantly improve the oxygen content lifetime after intravenous injection of oxygen microbubbles.
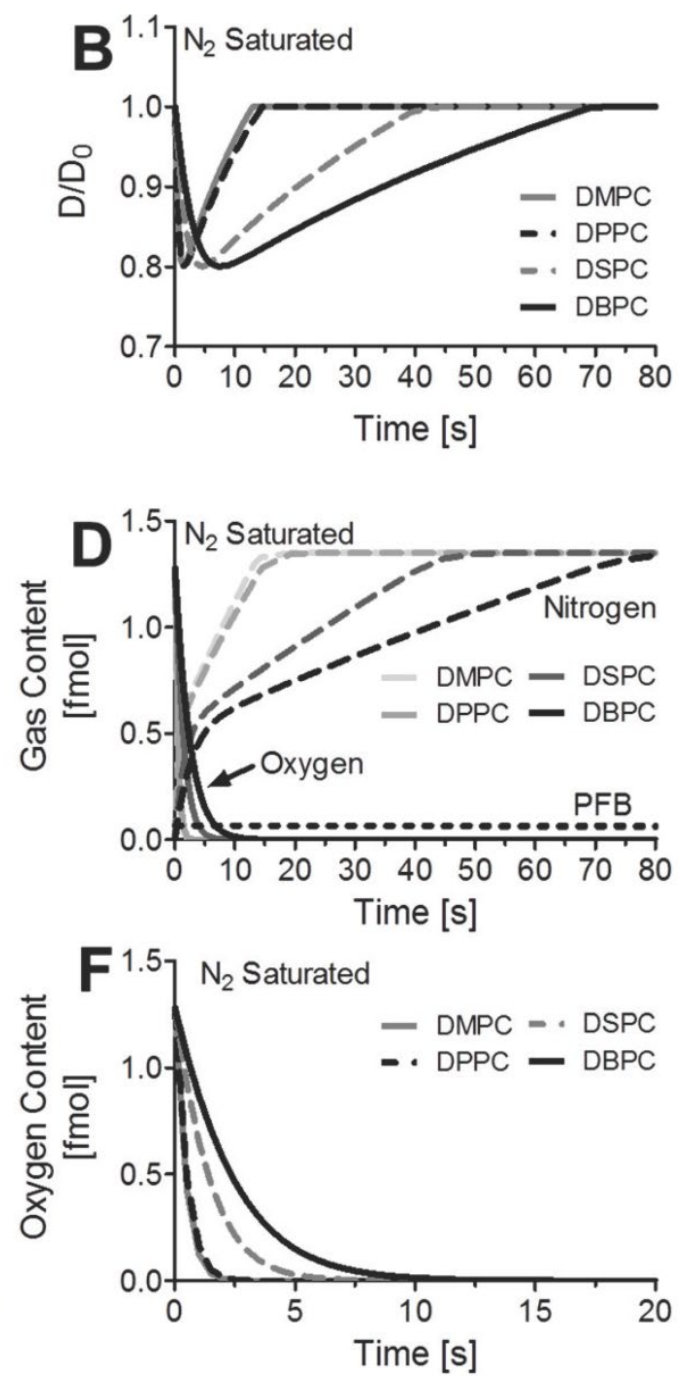

Figure 6. Simulations results for a 4- $\mathrm{mm}$ diameter microbubble coated with DMPC, DPPC, DSPC, or DBPC that initially is comprised of $95 \%$ oxygen and $5 \%$ perfluorobutane suddenly immersed in $A, C$ ) an air-saturated medium or $B, D$ ) a nitrogen-saturated medium at $22 \circ \mathrm{C}$ and I atm pressure. A-B) the diameter is normalized to the initial diameter and plotted as a function of time. C-D) the gas contents inside the microbubble over time. E-F) the oxygen content inside the microbubble over time. 
The simulations also predicted that each $4-\mu \mathrm{m}$ diameter bubble releases $\sim 1 \mathrm{fmol}$ of oxygen upon contact with the air-saturated medium, and it retains $\sim 0.25 \mathrm{fmol}$ of oxygen in the gas core. The simulation considers only a single microbubble, and not the ensemble where the partial pressure of oxygen in the bulk medium would increase as the microbubbles release their excess oxygen. For the experimental conditions, $\sim 3 \times 10^{9}$ microbubbles were injected into a $70 \mathrm{~mL}$ volume of water. The initial oxygen concentration for the air-saturated medium is estimated to be $285 \mathrm{nmol} / \mathrm{mL}$ based on Henry's constant shown in Table 1. According to the results of our simulation, we predict that the $300-\mu \mathrm{L}$ microbubble injection would raise the total oxygen concentration to $330 \mathrm{nmol} / \mathrm{mL}$ from the combined oxygen released from the microbubbles owing to natural diffusion $(43 \mathrm{nmol} / \mathrm{mL})$ and to dissolved oxygen present in the suspension medium ( $2 \mathrm{nmol} / \mathrm{mL}$ ). This corresponds to a $16 \%$ increase in dissolved oxygen by natural diffusion, which is higher than the measured $5 \%$ increase after $60 \mathrm{~s}$ of ultrasound (Fig. 2). The discrepancy between the theoretical and measured values for the increase in oxygen concentration may be due to the combined effects of the rise in bulk concentration of oxygen and the retardation of gas diffusion through the shell [40]. If all of the microbubbles were destroyed by ultrasound, then the oxygen content would be expected to rise by $19 \%$, which is more than the $15 \%$ increase observed experimentally after $240 \mathrm{~s}$ of ultrasound (Fig. 2 ). This difference may be due to incomplete microbubble destruction, for microbubbles that were not mixed into the region of ultrasound exposure, and the gradual loss of oxygen to the atmosphere.

\section{Discussion}

If cells in a tumor have mean oxygen partial pressure of approximately $3 \mathrm{mmHg}$ (as estimated for pancreatic, cervical and prostate cancer cells [9], and if the oxygen partial pressure is increased by $30 \mathrm{mmHg}$ via sufficient microbubbles delivered to those tumor cells and bursting the oxygen filled microbubbles by an ultrasound system, the OER will improve almost by a factor of 0.65 . This OER factor of 0.65 would mean a significant increase in the percentage of therapeutic efficiency according to the radiation survival curve. To convert the percent change in oxygen saturation to oxygen partial pressure $\left(\mathrm{pO}_{2}\right)$ we used the following formula:

Oxygen Saturation $(\%)=\left[\mathrm{pO}_{2} /(0.2094 \times 750 \mathrm{mmHg})\right] \times 100$

where 0.2094 is the mole fraction of oxygen in the atmosphere and $750 \mathrm{mmHg}$ is the absolute pressure. Ultimately, the amount of oxygen bubbles required to be injected to reach a desired oxygen partial pressure depends on the tumor size (e.g., measured by ultrasound) and its initial oxygen partial pressure (e.g., measured by electrodes and spectroscopic techniques). For example, to cause an increase of 42 $\mathrm{mmHg}$ of oxygen partial pressure in a tumor with a volume of $1 \mathrm{~mL}$, which corresponds to approximately $27 \%$ increase in oxygen saturation, $1 \mathrm{~mL}$ of microbubbles filled with oxygen mixture would need to be injected intravascularly assuming $1 \%$ of microbubbles would be destroyed in the tumor itself by an ultrasound system. The actual number of microbubbles destroyed in a tumor depends again on the tumor size, as well as the acoustic focus and circulation time for the microbubbles. However, for our assumption we consider a liver tumor that encompasses approximately $10 \%$ of the liver volume,[41] and we assume that the liver receives about $13 \%$ of circulatory blood flow.[42] Currently available microbubbles for imaging persist for several passes through the circulation,[43] so it is reasonable to expect that oxygen delivery bubbles could be designed similarly.

In order to develop a clinically viable microbubble oxygen delivery agent, one must be able to produce microbubbles in high yield. Early observations showed that pure oxygen microbubbles could be generated, but resulted in low yields and were generally not stable. The addition of perfluorobutane was shown to increase both the yield and stability of these oxygen microbubbles. The simulations showed that this increase in yield may have been an effect of the slower efflux of perfluorobutane gas compared to oxygen. This reduced efflux of gas increased the dissolution time by approximately 11 fold. As a result, there would be more time for lipids in the surrounding microbubble generating solution to adsorb onto the interface, decreasing the surface tension, halting the dissolution, and ultimately increasing the total yield of newly formed microbubbles.

Finally, the effect of administration of large quantities of microbubbles intravascularly in humans is unknown, and microbubbles are not yet approved for clinical therapeutics applications. The recommended dose of Definity, a microbubble used for contrast ultrasound imaging, is 10 microLiters per $\mathrm{kg}$. The maximum dose here $(300$ microLiters into $70 \mathrm{~mL}$ of water) was 33 fold greater than this clinically utilized dose (assuming $75 \mathrm{~mL}$ of blood per $\mathrm{kg}$ ). However, studies in rats have shown that doses 20 times that what is recommended for clinical imaging do not result in measurable changes in hepatic function, where much of the clearance occurs,[44] and there have been no adverse effects in repeated toxicology studies in 
rats and dogs with doses corresponding to 50 to 250 times the expected imaging dose in humans, respectively.[45]

In these experimental studies, we demonstrate that oxygen-carrier microbubbles can increase the oxygen saturation by diffusion and through ultrasound mediation. Without ultrasound, we showed that there was a marginal increase in oxygen saturation. Combining ultrasound and oxygen-carrier microbubbles, we can achieve an increase in local oxygen saturation that may be clinically relevant. Our results demonstrated that within 240 seconds, we can change the local oxygen concentration by a factor of 3 fold greater than that achieved in the same amount of time without ultrasound.

In our studies, we hypothesize that the $1 / 4$ " element ultrasound transducer, which only insonified a small percentage of our $70 \mathrm{~mL}$ sample volume, was the reason that complete microbubble destruction required 240 seconds. In-vivo, a phased array ultrasound system would be able to sonicate a tumor volume much more rapidly - releasing the majority of oxygen carriers almost instantaneously. The combination of the imaging capabilities of phased array ultrasound systems will additionally allow registration of the area to be treated.

In the in-vivo case, however, the limiting factor would likely be the amount of carriers that are present in the tumor vasculature. Administration of the oxygen carrier microbubbles in a feeder artery close to the tumor site in conjunction with ultrasound would greatly enhance local delivery, in contrast to peripheral vein injection typically used with the administration of microbubble-type contrast agents.

Ultrasound is a widespread, safe, portable, and relatively inexpensive modality. Ultrasound can be used to not only image the tumor target, but also to apply energy to mediate the therapeutic effect of oxygen delivery. A system to apply ultrasound during radiotherapy is technologically feasible [46]. Based on our initial studies, the ultrasound parameters to mediate tumor oxygenation through acoustically-active oxygen delivery vehicles will likely be within the FDA limits already approved for clinical ultrasound imaging.

\section{Conclusions}

We give an explanation for the high yield and stability of oxygen-perfluorobutane microbubbles and demonstrate preliminary results of the first study of oxygen delivery via stabilized microbubbles in conjunction with ultrasound. We also hypothesize that this method will provide a minimally-invasive technology which will allow a physician to locally deliver oxygen to tumor tissue during radiation treatment and enhance tumor radiation sensitivity. This novel approach has the potential to substantially improve therapeutic treatment for cancer by both increasing tumor response to radiation, and reducing the radiation dose required for a therapeutic effect.

\section{Competing Interests}

The authors have declared that no competing interest exists.

\section{References}

1. Carmeliet P, Jain RK. Principles and mechanisms of vessel normalization for cancer and other angiogenic diseases. Nature reviews. 2011;10: 417-27.

2. Sorg BS, Moeller BJ, Donovan O, Cao Y, Dewhirst MW. Hyperspectral imaging of hemoglobin saturation in tumor microvasculature and tumor hypoxia development. J Biomed Opt. 2005; 10: 44004.

3. Troost EG, Laverman P, Kaanders JH, Philippens M, Lok J, Oyen WJ, et al. Imaging hypoxia after oxygenation-modification: comparing [18F]FMISO autoradiography with pimonidazole immunohistochemistry in human xenograft tumors. Radiother Oncol. 2006; 80: 157-64.

4. Hedley D, Pintilie M, Woo J, Morrison A, Birle D, Fyles A, et al. Carbonic anhydrase IX expression, hypoxia, and prognosis in patients with uterine cervical carcinomas. Clin Cancer Res. 2003; 9: 5666-74.

5. Nordsmark M, Bentzen SrM, Rudat V, Brizel D, Lartigau E, Stadler P, et al. Prognostic value of tumor oxygenation in 397 head and neck tumors after primary radiation therapy. An international multi-center study. Radiotherapy and Oncology. 2005; 77: 18-24.

6. Nordsmark M, Loncaster J, Aquino-Parsons C, Chou SC, Ladekarl M, Havsteen $\mathrm{H}$, et al. Measurements of hypoxia using pimonidazole and polarographic oxygen-sensitive electrodes in human cervix carcinomas. Radiother Oncol. 2003; 67: 35-44.

7. Nordsmark M, Overgaard J. A confirmatory prognostic study on oxygenation status and loco-regional control in advanced head and neck squamous cell carcinoma treated by radiation therapy. Radiother Oncol. 2000; 57: 39-43.

8. Kaanders JH, Bussink J, van der Kogel AJ. Clinical studies of hypoxia modification in radiotherapy. Semin Radiat Oncol. 2004; 14: 233-40.

9. Brown JM, Wilson WR. Exploiting tumour hypoxia in cancer treatment. Nat Rev Cancer. 2004; 4: 437-47.

10. Hall EJ. Radiation, the Two-Edged Sword: Cancer Risks at High and Low Doses. Cancer Journal. 2000; 6: 343-50.

11. Brown JM. Tumor hypoxia in cancer therapy. Methods Enzymol. 2007; 435: 297-321.

12. Hodgkiss RJ, Roberts IJ, Watts ME, Woodcock M. Rapid-mixing Studies of Radiosensitivity with Thiol-depleted Mammalian Cells. International Journal of Radiation Biology. 1987; 52: 735-44.

13. Diehn M, Cho RW, Lobo NA, Kalisky T, Dorie MJ, Kulp AN, et al. Association of reactive oxygen species levels and radioresistance in cancer stem cells. Nature. 2009; 458: 780-3.

14. Wilson SR, Burns PN. Microbubble-enhanced US in body imaging: what role? Radiology. 2010; 257: 24-39.

15. Wilson SR, Greenbaum LD, Goldberg BB. Contrast-enhanced ultrasound: what is the evidence and what are the obstacles? AJR Am J Roentgenol. 2009; 193: 55-60.

16. Gessner R, Dayton PA. Advances in molecular imaging with ultrasound. Molecular imaging. 2010; 9: 117-27.

17. Sirsi SR, Flexman ML, Vlachos F, Huang J, Hernandez SL, Kim HK, et al. Contrast Ultrasound Imaging for Identification of Early Responder Tumor Models to Anti-Angiogenic Therapy. Ultrasound in Med. Biol. 2012; 38: 1019-29.

18. Streeter JE, Gessner RC, Tsuruta J, Feingold S, Dayton PA. Assessment of Molecular Imaging of Angiogenesis with Three-Dimensional Ultrasonography. Molecular imaging. 2011; 10(6):460-8.

19. Gerber F, Waton G, Krafft MP, Vandamme TF. Long lived microbubbles for oxygen delivery. Artif Cells Blood Substit Immobil Biotechnol. 2007; 35: 119-24.

20. Lundgren C, Bergoe G, Olszowka A, Tyssebotn I. Tissue nitrogen elimination in oxygen-breathing pigs is enhanced by fluorocarbon-derived intravascular micro-bubbles. Undersea Hyperb Med. 2005; 32: 215-26. 
21. Lundgren CE, Bergoe GW, Tyssebotn IM. Intravascular fluorocarbon-stabilized microbubbles protect against fatal anemia in rats. Artif Cells Blood Substit Immobil Biotechnol. 2006; 34: 473-86.

22. Swanson EJ, Borden MA. Injectable oxygen delivery based on protein-shelled microbubbles. . Nano Life. 2010; 1: 215-8.

23. Swanson EJ, Mohan V, Kheir J, Borden MA. Phospholipid-stabilized microbubble foam for injectable oxygen delivery. Langmuir. 2010; 26: 15726-9.

24. Burkard ME, Van Liew HD. Oxygen transport to tissue by persistent bubbles: theory and simulations. J Appl Physiol. 1994; 77: 2874-8.

25. Van Liew HD, Burkard ME. High oxygen partial pressure in tissue delivered by stabilized microbubbles. Theory. Adv Exp Med Biol. 1997; 411: 395-401.

26. Kwan JJ, Borden MA. Microbubble dissolution in a multigas environment. Langmuir. 2010; 26: 6542-8.

27. Kwan JJ, Borden MA. Lipid monolayer dilatational mechanics during microbubble gas exchange. Soft Matter. 2012; 8: 4756-4766.

28. Borden MA, Martinez GV, Ricker J, Tsvetkova N, Longo M, Gillies RJ, et al. Lateral phase separation in lipid-coated microbubbles. Langmuir. 2006; 22: 4291-7.

29. Chomas JE, Dayton P, May D, Ferrara K. Threshold of fragmentation for ultrasonic contrast agents. J Biomed Opt. 2001; 6: 141-50.

30. Kabalnov A, Klein D, Pelura T, Schutt E, Weers J. Dissolution of multicomponent microbubbles in the bloodstream: 1. Theory. Ultrasound Med Biol. 1998; 24: 739-49.

31. Wilhelm E, Battino R, Wilcock RJ. Low-pressure solubility of gases in liquid water. Chemical Reviews. 1977; 77: 219-62.

32. Himmelblau DM. Diffusion of Dissolved Gases in Liquids. Chemical Reviews. 1964; 64: 527-50.

33. Lide D. CRC Handbook of Chemistry and Physics, 89th Edition (CRC Handbook of Chemistry \& Physics). CRC Press; 2009.

34. Tee LS, Gotoh S, Stewart WE. Molecular Parameters for Normal Fluids. Lennard-Jones 12-6 Potential. Industrial \& Engineering Chemistry Fundamentals. 1966; 5: 356-63.

35. Taylor P. Ostwald ripening in emulsions. Advances in Colloid and Interface Science. 1998; 75: 107-63.

36. Kabalnov A. Ostwald Ripening and Related Phenomena. Journal of Dispersion Science and Technology. 2001; 22: 1-12.

37. Duncan PB, Needham D. Test of the Epstein-Plesset model for gas microparticle dissolution in aqueous media: effect of surface tension and gas undersaturation in solution. Langmuir. 2004; 20: 2567-78.

38. Kim DH, Costello MJ, Duncan PB, Needham D. Mechanical Properties and Microstructure of Polycrystalline Phospholipid Monolayer Shells: Novel Solid Microparticles. Langmuir. 2003; 19: 8455-66.

39. Dressaire E, Bee R, Bell DC, Lips A, Stone HA. Interfacial polygonal nanopatterning of stable microbubbles. Science (New York, NY. 2008; 320: 1198-201.

40. Borden MA, Longo ML. Oxygen Permeability of Fully Condensed Lipid Monolayers. The Journal of Physical Chemistry B. 2004; 108: 6009-16.

41. Ettinger DS, Leichner PK, Siegelman SS, Fishman EK, Klein JL, Order SE. Computed tomography assisted volumetric analysis of primary liver tumor as a measure of response to therapy. American journal of clinical oncology. 1985; 8: 413-8.

42. Miller KR, Levine JS. Biology. Upper Saddle River: Prentice Hall; 2002.

43. Mullin L, Gessner R, Kwan J, Kaya M, Borden MA, Dayton PA. Effect of anesthesia carrier gas on in vivo circulation times of ultrasound microbubble contrast agents in rats. Contrast media \& molecular imaging. 2011; 6: 126-31.

44. Kindberg GM, Tolleshaug H, Roos N, Skotland T. Hepatic clearance of Sonazoid perfluorobutane microbubbles by Kupffer cells does not reduce the ability of liver to phagocytose or degrade albumin microspheres. Cell Tissue Res. 2003; 312: 49-54.

45. Schneider M, Anantharam B, Arditi M, Bokor D, Broillet A, Bussat P, et al. BR38, a new ultrasound blood pool agent. Investigative radiology. 2010; 46: 486-94.

46. Schlosser J, Salisbury K, Hristov D. Telerobotic system concept for real-time soft-tissue imaging during radiotherapy beam delivery. Medical physics. 2010; 37: 6357-67.

\section{Appendix}

The dissolved oxygen concentration in water is $8.8 \mathrm{mg} / \mathrm{L}$ at a temperature of 22 degrees Celsius and an atmospheric pressure of $760 \mathrm{mmHg}$. The solubility of oxygen changes with total atmospheric pressure and the temperature as described by Henry's Law. Our experimental conditions stayed the same during the oxygen saturation measurements at a temperature of 22 degrees Celsius and atmospheric pressure of 760 $\mathrm{mmHg}$. The number of microbubbles per milliliter of microbubble solution and the average size of microbubbles were used to calculate the total volume of oxygen contained in the microbubbles infused into the water using the following equation:

$$
V_{O_{2}}=C_{\text {bubble }} \times \frac{4}{3} \pi r^{3}
$$

where, $V_{O_{2}}$ is the volume of oxygen in solution, $C_{\text {bubble }}$ is the number of microbubbles per $\mathrm{mL}$, and $r$ is the average radius of the microbubbles. 